\title{
Ribotipificación de aislamientos de Mannheimia haemoly tica serotipo 1 obtenidos de exudado nasal de bovinos productores de leche en México
}

\section{Ribotyping of isolates of Mannheimia haemolytica serotype 1 obtained from nasal exudate of dairy cattle in Mexico}

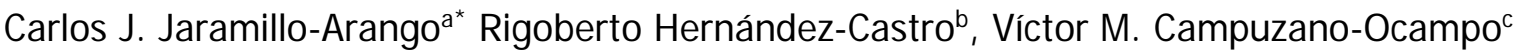 \\ Gabriela Delgado-Sapién ${ }^{d}$, Rosario Morales-Espinosad , J uan Xicohtencatl-Cortése , \\ Francisco Suárez-Güemes ${ }^{f}$, Francisco Trigo-Taverag
}

\begin{abstract}
RESUMEN
Se realizó la caracterización genética de 106 aislamientos de Mannheimia haemolytica serotipo 1 (S1) obtenidos de exudado nasal de bovinos clínicamente sanos (BCS) $(n=80)$ y enfermos (BCE) $(n=26)$ de neumonía de dos granjas lecheras del centro y norte de México, mediante la técnica de ribotipificación. De los cuales se extrajo el DNA para realizar el proceso de digestión con la endonucleasa de restricción Hindl II y la posterior ribotipificación, que se realizó utilizando una sonda que contenía el operon rrnB rRNA. Se identificaron dos patrones de ribotipos: Rt1 y Rt2, en ambos casos, con bandas de hibridación con tamaños aproximados entre 0.78 y $19.70 \mathrm{~kb}$. El Rt1 presentó 11 bandas de hibridación y el Rt2 13. El $96 \%$ de los aislamientos (102/ 106) se agruparon en un cluster dentro del Rt1. Entre el Rt1 y el Rt2 se presentó un valor de similitud de $70 \%$. No se identificaron diferencias entre los Rt de los aislamientos de los animales BCS o BCE. Estos resultados indican que la mayoría de las cepas se agrupan dentro un mismo Rt (Rt1) conformando un solo cluster, independientemente del origen de las mismas y del estado de salud.
\end{abstract}

PALABRAS CLAVE: Mannheimia, Ribotipos, Bovinos, Exudado nasal.

\begin{abstract}
One hundred and six (106) isolates of Mannheimia haemolytica serotype 1 (S1) obtained from nasal exudates of clinically healthy cows $(n=80)$ and cows diagnosed with pneumonia $(n=26)$ from two dainy farms located in central and northern Mexico were genetic characterized using a ribotyping technique. DNA was digested by Hind II and a probe containing the rrnB rRNA operon was used for ribotyping. Two ribotyping patterns were identified: Rt1 and Rt2, both with bands of approximate size between 0.78 and $19.70 \mathrm{~kb}$. Rt1 had 11 hybridization bands and Rt2, 13. Ninety-six (96) percent of the isolates (102/ 106) were grouped into a cluster within Rt1; the similarity value between Rt1, Rt2 and the reference strain was $70 \%$. There were no differences between Rt isolates from healthy cows or cows with pneumonia. These results show that the majority of the strains were grouped within the Rt1 forming a single cluster, regardless of their geographic origin or the cows' health status.
\end{abstract}

KEY WORDS: Mannheimia, Ribotypes, Dairy cows, Nasal exudate.

Recibido el 26 de enero de 2016. Aceptado el 21 de marzo de 2016.

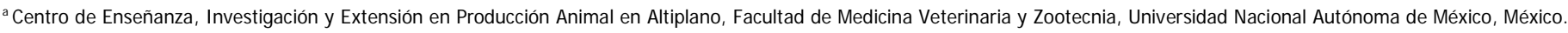

' Hospital General “Dr. Manuel Gea González", Dirección de Investigación, Secretaría de Salud. México.

c Departamento de Medicina Preventiva y Salud Pública, Facultad de Medicina Veterinaria y Zootecnia, Universidad Nacional Autónoma de México. México.

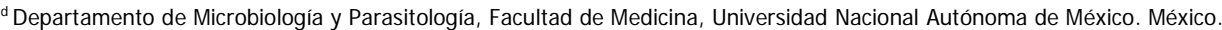

e Hospital Infantil de México “Dr. Federico Gómez", Secretaría de Salud, México.

f Departamento de Microbiología e Inmunología, Facultad de Medicina Veterinaria y Zootecnia, Universidad Nacional Autónoma de México. México.

g Departamento de Patología, Facultad de Medicina Veterinaria y Zootecnia, Universidad Nacional Autónoma de México.

* Autor de correspondencia: cjja@unam.mx. 
Anteriormente Pasteurella haemolytica se clasificaba en dos biotipos, A y T de acuerdo a su habilidad para fermentar la arabinosa o latrehalosa, respectivamente. El complejo $P$. haemolytica negativo a la trehalosa fue reclasificado hace más de dos décadas dentro del género Mannheimia que incluye al menos seis especies: $M$. haemolytica, $M$. granulomatis, $M$. varigena, $M$. ruminalis, $M$. caviae y M. glucosida. Conforme a la clasificación actual, $M$. haemolytica incluye los serotipos capsulares 1 , 2, 5-9, 12-14, 16 y 17 de $P$. haemolyticd ${ }^{(1,2)}$.

M. haemolytica (Mh) es la bacteria más patógena dentro del género, y frecuentemente asociada con enfermedades del aparato respiratorio de los bovinos, particularmente con la aún definida pasteurelosis neumónica bovina, también conocida como fiebre de embarque ${ }^{(3)}$. Mh reside en las tonsilas y la nasofaringe de animales aparentemente sanos $^{(4,5)}$, pero en animales inmunocomprometidos por infecciones virales preexistentes o estresados por el manejo, principalmente menores de un año o recientemente transportados, puede descender a los pulmones y desarrollar una neumonía ${ }^{(3,6)}$. La morbilidad y la mortalidad asociada con esta enfermedad producen grandes pérdidas, por lo que se considera la causa más relevante en cuanto a las pérdidas económicas en la industria bovina ${ }^{(3,5)}$.

Los estudios sobre caracterización epidemiológica requieren del uso tanto de métodos fenotípicos como genotípicos. Los métodos fenotípicos para la caracterización de las especies de Mannheimia se han utilizado durante mucho tiempo y aunque se acepta que su reproducibilidad es alta, sus limitaciones han sido reconocidas ampliamente ${ }^{(7,8)}$. En estudios realizados para evaluar la especificidad de la serotipificación como una herramienta diagnóstica, se ha demostrado que no es un método confiable para la correcta identificación de Mh, y se hace énfasis sobre la necesidad de una amplia caracterización fenotípica y genotípica para la adecuada identificación de este microorganismo, considerando las dificultades que han presentado otros estudios para su clasificación basado solamente en la fenotipificación y la serotipificación ${ }^{(2,8)}$, teniendo en cuenta que el género Mannheimia abarca taxones de una gran heterogeneidad fenotípica y genotípica(8).
Entre los métodos de tipificación molecular se cuenta con diversas técnicas, entre las cuales se pueden destacar la hibridación de ADN-ADN, electroforesis de enzimas multilocus (EEML), amplificación al azar del polimorfismo del ADN (RAPD), electroforesis en gel de campo pulsado (PFGE), y el análisis de restricción para la detección de los genes del ARNr o ribotipificación. Esta última es una herramienta que ha demostrado ser de gran utilidad en estudios de epidemiología molecular de diferentes especies bacterianas ${ }^{(9,10)}$ y estudios que involucran a Pasteurellad ${ }^{(6-8)}$.

En México no se han realizado estudios de caracterización genómica de aislamientos de Mannheimia obtenidas de bovinos. Los estudios realizados desde la década de los 80 se han enfocado en la caracterización fenotípica del microorganismo, los cuales reportan a los serotipos A1 y A2 como los más frecuentes en bovinos ${ }^{(11,12)}$. En este estudio, se utilizó la ribotipificación con el propósito de determinar las características y las diferencias genotípicas de aislamientos de Mannheimia haemolytica serotipo 1 (S1), obtenidos de exudado nasal de bovinos clínicamente sanos (BCS) y clínicamente enfermos (BCE) de neumonía, de granjas lecheras ubicadas en dos zonas ganaderas de gran importancia en el país.

Se emplearon 106 aislamientos de Mannheimia haemolytica serotipo 1 (S1) obtenidos de exudado nasal de bovinos clínicamente sanos (BCS) $(n=80)$ y clínicamente enfermos de neumonía (BCE) $(n=26)$ de dos complejos lecheros; uno ubicado en el valle central de Tizayuca $(T Z Y) \quad(B C S=29 ; B C E=18)$, estado de Hidalgo y otro en la Región Lagunera de Torreón (TOR) $(B C S=51 ; B C E=8)$ en el estado de Coahuila, México. El aislamiento, identificación y serotipificación se realizaron en estudios previos mediante métodos convencionales de cultivo in vitro y pruebas bioquímicas e inmunológicas ${ }^{(13,14)}$. Además, se incluyeron las cepas de referencia de Mh de lo serotipos 1, 2, 5-9, 11, 12 (donadas por el Dr. GH Frank y el Dr. B Briggs, NADC, USDA).

Los aislamientos se crecieron en caldo infusión cerebro corazón (BHI) durante $18 \mathrm{~h}$ a $37{ }^{\circ} \mathrm{C}$ con agitación orbital a $250 \mathrm{rpm}$. La extracción del ADN cromosómico se realizó según el método descrito por Pitcher et $a{ }^{(15)}$. La digestión del DNA se realizó 
utilizando la enzima de restricción HindlII (Invitrogen ${ }^{\circledR}$ ) a $37{ }^{\circ} \mathrm{C}$ durante toda la noche, siguiendo las instrucciones del fabricante.

Los productos de las digestiones se separaron por electroforesis en geles de agarosa al $1 \%$ teñidos con bromuro de etidio a $20 \mathrm{~V}$ por $19 \mathrm{~h}$. El DNA se transfirió a membranas de nylon $(0.45 \mu \mathrm{m})$ mediante el método de transferencia capilar (Figura 1).

La sonda que contiene el operón rmB rRNA de E. coli se obtuvo del plásmido pKK3535(16). Brevemente, la extracción del ADN plasmídico pKK3535 se realizó utilizando el sistema comercial

Figura 1. Perfil electroforético del ADN cromosómico de cepas de $M$. haemolytica de referencia y de aislamientos de campo digerido con HindIII en gel de agarosa al $1 \%$ en TBE $0.5 X$, teñido con bromuro de etidio. Líneas 1 a 7 cepas de referencia serotipos 1, 2, 5-9, líneas 8 y 9 aislamientos de campo.

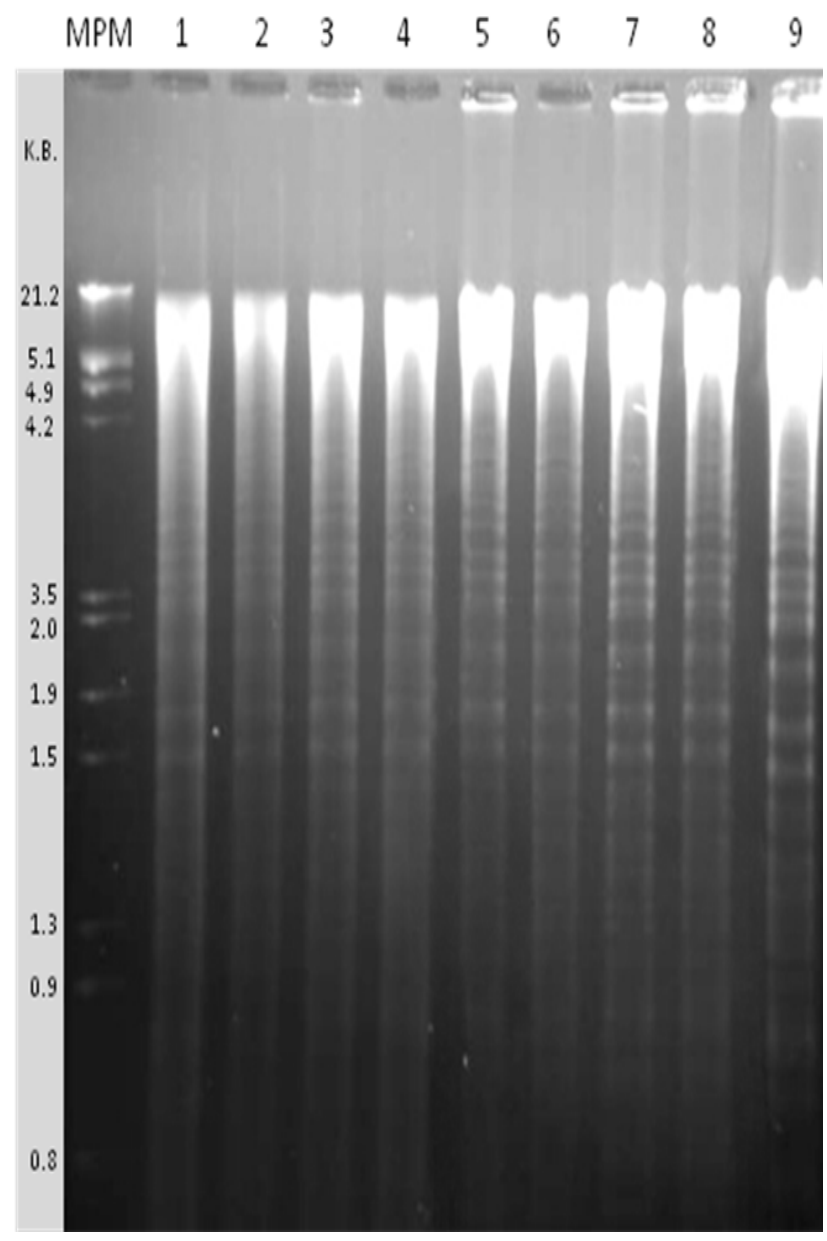

QIAprep (Qiagen ${ }^{\circledR}$ ), el ADN obtenido se digirió con la enzima de restricción BCA (New England BioLabs ${ }^{\circledR}$ ) de acuerdo con las instrucciones del fabricante. El producto de $5.9 \mathrm{~kb}$ que contiene el operón $r r n B$ se purificó utilizando el sistema comercial QiaEX II $\left(\right.$ Qiagen $\left.^{\circledR}\right)$ y posteriormente se marcó con digoxigenina utilizando el sistema DIGHigh Prime $\left(\right.$ Roche $\left.^{\circledR}\right)$.

Las membranas de nylon se colocaron en una solución de prehibridación a $42{ }^{\circ} \mathrm{C}$ por $2 \mathrm{~h}$. Posteriormente se depositaron en una solución de hibridación que contenía la sonda marcada a $42{ }^{\circ} \mathrm{C}$ por $16 \mathrm{~h}$ (durante la noche). Las membranas se bloquearon durante $1 \mathrm{~h}$ y posteriormente se agregó el conjugado anti DIG-AP diluido 1:20,000 en buffer maléico y las membranas se incubaron por $30 \mathrm{~min}$. Para la detección de las bandas las membranas se cubrieron con $1 \mathrm{ml}$ de reactivo iniciador (CDP Starter-CSPD, Roche ${ }^{\circledR}$ ) y se expusieron a películas auto-radiográficas (Hyperfilm, Amersham Biosciences).

Los patrones de los ribotipos se analizaron mediante el programa BioNumerics versión 7.0 (Applied Maths). Se elaboraron dendrogramas para analizar la similitud entre los aislamientos y las cepas de referencia de $M$. haemolytica mediante el coeficiente de Dice con máxima similitud. El análisis de los cluster se realizó mediante el uso del "Unweighted Pair Group Method of Arithmetic Averages" (UPGMA).

En los 106 aislamientos se identificaron dos patrones de ribotipos: Rt1 y Rt2; el Rt1 con 11 bandas de hibridación y el Rt2 con 13 bandas, en ambos casos con tamaños aproximados de 0.78 a $19.70 \mathrm{~kb}$ (Figura 2). El $96 \%(102 / 106)$ de los aislamientos fue Rt1 (BCS= 77; $B C E=25)$, el cual presentó bandas de hibridación con tamaños aproximados de $0.78,1.53,1.92,2.03,2.9,3.27$, $4.77,8.05,11.80,14.90$ y $19.70 \mathrm{~kb}$; cuatro aislamientos fueron Rt2 $(\mathrm{BCS}=3 ; \mathrm{BCE}=1)$. Los dos Rt compartieron las bandas de $0.78,1.53$, 2.9, 3.27, $4.77,8.05,11.8,14.9$ y $19.7 \mathrm{~kb}$; las bandas de 1.92 y $2.03 \mathrm{~kb}$ presentes en el Rtl estaban ausentes en el RT2; y las bandas de 1.0, 1.02, 1.7, 3.7 y $4.2 \mathrm{~kb}$ del Rt2 estaban ausentes en el Rt1. En comparación con las cepas de referencia ambos Rt ( 1 y 2) sólo compartieron nueve bandas de $0.78,1.53,2.9,3.27$, 
$4.77,8.05,11.8,14.9$ y $19.7 \mathrm{~kb}$. Por su parte el Rt2 compartió las bandas de 1.0, 1.02 y $3.7 \mathrm{~kb}$.

La relación genética entre los 2 Rt se presenta en el dendrograma de la Figura 1, donde se muestran las estimaciones del grado de similitud entre los aislamientos de campo y las cepas de referencia de Mh. No se identificaron diferencias entre los Rt de los aislamientos de animales BCS o BCE.

En el dendrograma se observan los tres cluster que se conformaron, cada uno con $100 \%$ de similitud. En el primero se agrupan lo serotipos de la cepa de referencia de Mh, y todos ellos presentaron el mismo patrón de bandas de hibridación. En el segundo cluster se agrupan los aislamientos del Rt2, tres aislamientos de TZY y uno de TOR, y en el tercer cluster una muestra conformada por 12 aislamientos del Rt1, seis de TZY y seis de TOR. Se observaron los siguientes porcentajes de similitud: $91 \%$ entre el Rt2 y la cepa de referencia y entre el Rt1 y la cepa de referencia $70 \%$ (Figura 2 ).

La mayoría de los estudios sobre Mh realizados en México se han enfocado en la caracterización

Figura 2. Principales ribotipos presentes en cepas de $M$. haemolytica A1 usando la enzima de restricción HindIII. Ref= cepas de referencia de diferentes serotipos de $M$. haemolytica; Rt1= perfil del ribotipo R1; Rt2= perfil del ribotipo R2; TZY= Tizayuca; TOR= Torreón

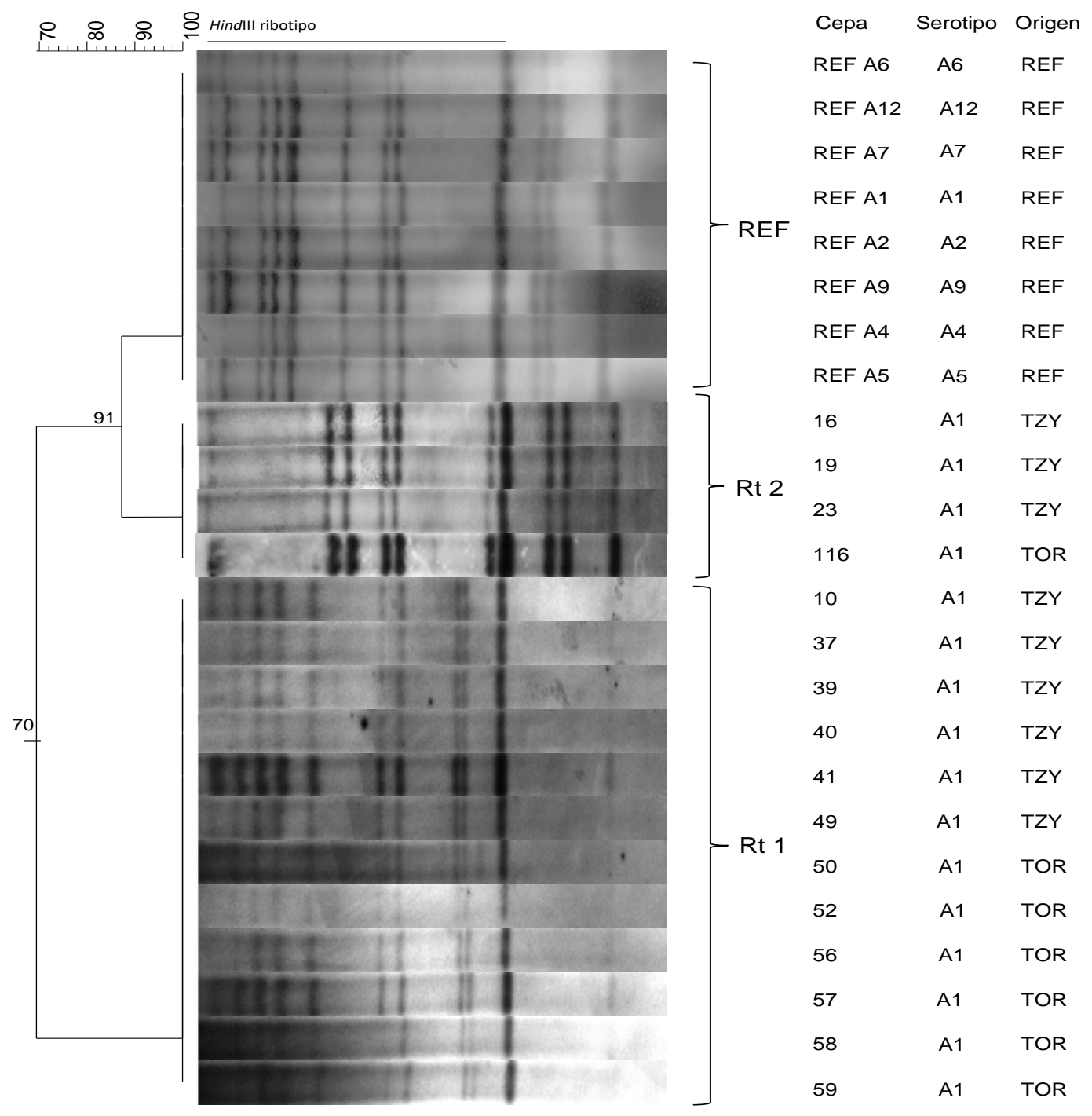


fenotípica del microorganismo; en el presente estudio la caracterización de aislamientos de Mannheimia haemolytica obtenidos de exudado nasal de bovinos, se confirmó mediante la ribotipificación con base en resultados de estudios taxonómicos previos ${ }^{(13,14)}$.

En este estudio la ribotipificación con la enzima HindIII pudo diferenciar el S1 de M. haemolytica en dos grupos genéticos diferentes (Rt1 y Rt2), y demostró ser de gran utilidad en la caracterización de aislamientos de Mannheimia spp., esto coincide con lo reportado por otros estudios ${ }^{(8,17,18)}$ pero contrasta con los hallazgos de Murphy et $a^{\left({ }^{6}\right)}$, quienes reportaron que la enzima fue incapaz de distinguir ribotipos entre aislamientos de $P$. haemolytica S1.

Tanto en TZY como en TOR la mayoría de los aislamientos $\mathrm{S} 1$ se agruparon en el Rt1 en un cluster totalmente homogéneo, lo cual coincide con lo reportado por Kodjo et a ${ }^{(17)}$ quienes caracterizaron cepas de $P$. haemolytica ( $M$. haemolytica) utilizando la enzima de restricción HindllI. Estos hallazgos evidencian que la mayoría de los aislamientos de Mh S1, de TZY y de TOR, fueron genéticamente indistinguibles, ya que dentro de los cluster correspondientes compartieron el mismo ribotipo con el mismo número de bandas, además no hubo diferencias entre los Rt aislados de los BCS y BCE. Desde el punto de vista epidemiológico se puede considerar que estos aislamientos corresponden a una misma cepa dentro de cada uno de los cluster que agrupan el S1. Los aislamientos del Rt2 presentaron una estrecha relación genética con las cepas de referencia de Mh (91\%); sin embargo, el Rt1 mostró una similitud del $70 \%$ con dichas cepas. Estas diferencias en el patrón de bandas entre las cepas de referencia y las correspondientes a los Rt 1 y 2 podrían deberse a mutaciones en el genoma de estas últimas, como lo señala Snipes et $a\left(^{(19)}\right.$, quizá como consecuencia de influencias del medio ambiente, muchas de las cuales son desconocidas; un ejemplo de ello es la variación fenotípica por influencia del ambiente externo de las proteínas de membrana externa reguladas por hierro en $P$. multocida. Asimismo, es posible que dicha diferencia tenga como base la ausencia o presencia de sitios de corte de la enzima de restricción utilizada. Un solo cambio de nucleótido puede cambiar un sitio y generar un cluster diferente. En este trabajo se utilizaron cepas de referencia de los años 19701980, que al compararlas con los aislamientos obtenidos (2003) se observaron estos cambios. El encontrar un grupo homogéneo nos demuestra que estos aislamientos están sometidos a una presión de selección constante, donde se observa un patrón de ribotipos altamente homogéneo. Asimismo, el encontrar un grupo homogéneo demuestra que los aislamientos analizados en este trabajo forman un núcleo genéticamente estable en nuestro país, al menos de los aislamientos analizados.

En este estudio se encontraron dos Rt que correspondieron a un solo serotipo, lo cual coincide con lo reportado por Snipes et $a^{(20)}$. Estos aislamientos del mismo serotipo, pero de diferente Rt pueden ser cepas con diferente genotipo que posean porciones de sus genomas que codifiquen en la producción de ciertos antígenos similares que les permiten compartir el serotipo, pero no el genotipo, ya que poseen otras porciones significativas del genoma que son diferentes, por ejemplo, genes rRNA que son altamente conservados y no son sujetos de mutaciones frecuentes ${ }^{(19)}$.

No obstante que se ha cuestionado el valor de la ribotipificación para calcular las distancias taxonómicas entre cepas o para la diferenciación de aislamientos ${ }^{(21)}$, esta herramienta ha demostrado su validez para la clasificación y agrupación de diversos grupos de bacterias, incluyendo la familia Pasteurellaceae ${ }^{(18)}$. Se ha podido demostrar la correlación entre los dusters obtenidos por ribotipos y mediante electroforesis de enzimas multilocus (MLEE) tanto de $P$. multocida como de M. haemolyticd ${ }^{22,23)}$, así como con el análisis por endonucleasas de restricción (REA) de P. multocidd ${ }^{(19)}$.

De acuerdo con los hallazgos de este trabajo, la ribotipificación fue útil para distinguir aislamientos del mismo género y especie, en coincidencia con los resultados de otros estudios con Mannheimid ${ }^{(7,20,24)}$, o con otras bacterias de la familia Pasteurellaceae ${ }^{(19,24,25)}$, concluyendo que la mayoría de los aislamientos se agrupan dentro un mismo Rt (Rt1) conformando un solo cluster, independientemente del origen de las mismas y del estado de salud de los animales. 


\section{AGRADECIMIENTOS}

Este estudio fue financiado por el CONACyT (Proyecto G38590-B). Se agradece a los ganaderos de las Cuencas Lecheras de Tizayuca, Hidalgo y le Comarca Lagunera, Torreón, Coahuila, así como al Departamento de Microbiología e Inmunología de la Facultad de Medicina Veterinaria y Zootecnia de la UNAM, por el apoyo y las facilidades otorgadas para la realización de este trabajo. A los Dres. G. H. Frank y B. Briggs por la donación de las cepas de referencia de M. haemolytica.

\section{LITERATURA CITADA}

1. Angen O, Mutters R, Caugant DA, Olsen JE, Bisgaard M. Taxonomic relationships of the [ Pasteurella] haemolytica complex as evaluated by DNA-DNA hybridizations and 16SrRNA sequencing with proposal of Mannheimia haemolytica gen., comb.nov., Mannheimia granulomatis comb. nov., Mannheimia glucosida sp. nov., Mannheimia ruminalis sp. nov. and Mannheimia varigena sp. nov. Int J Syst Bacteriol 1999;49:67-86.

2. Angen O, Quirie M, Donachie W, Bisgaard M. Investigations on the species specificity of Mannheimia (Pasteurella) haemolytica serotyping. Vet Microbiol 1999;65:283-290.

3. Highlander SK. Molecular genetic analysis of virulence in Mannheimia. Frontiers in Bioscience 2001;6:1128-1150.

4. Rowe HA, Poxton IR, Donachie W. Survival of Mannheimia (Pasteurella) haemolytica in tracheobronchial washings of sheep and cattle. Vet Microbiol 2001;81:305-314.

5. Narayanan SK, Nagaraja TG, Chengappa MM, Stewart GC. Leukotoxins of gram-negative bacteria. Vet Microbiol 2002:84:337356.

6. Murphy GL, Robinson LC, Burrows GE. Restriction endonuclease analysis and ribotyping differentiate Pasteurella haemolytica serotype Al isolates from cattle within a feedlot. Clin Microbiol 1993; 31(9):2303-2308.

7. Chaslus-Dancla E, Lesage-Descauses MC, Leroy-Sétrin S, Martel J L, Courdet P, Lafont JP. Validation of random amplified polymorphic DNA assays for ribotyping as tools for epidemiological surveys of Pasteurella from animals. Vet Microbiol 1996;52:91-102.

8. Angen O, Ahrens P Bisgaard, M. Phenotypic and genotypic characterization of Mannheimia (Pasteurella) haemolyticalike strains isolated from diseased animals in Denmark. Vet Microbiol 2002; 84:103-114.

9. Blumberg HM, Stephens DS, Licitra C, Pigott N, Facklam R, Swaminathan B, Wachsmuth IK. Molecular epidemiology of group B Streptococcal infections: use of restriction endonuclease analysis of chromosomal DNA and DNA Restriction Fragment Length Polymorphisms of ribosomal RNA genes (Ribotyping). J Infect Dis 1992; 166:574-759.

10. Christensen H, Dziva F, Olsen JE, Bisgaard M. Genotypic heterogneity of Pasteurella gallinarum as shown by ribotyping and 16S rRNA sequencing. Avian Pathol 2002;31:603-610.
11. Blanco-Viera FJ, Trigo FJ, Jaramillo-Meza L, Aguilar-Romero F. Serotypes of Pasteurella multocida and Pasteurella haemolytica isolated from pneumonic lesions in cattle and sheep from Mexico. Rev Lat-Am Microbiol 1995; 37: 121-126.

12. Pijoan P, Aguilar RF, Morales AF. Caracterización de los procesos neumónicos en becerros lecheros de la región de Tijuana, Baja California, México. Vet Méx 1999; 30(2):149-155.

13. Jaramillo-Arango Cl, Hernández-Castro R, Suárez-Güemes F, Martínez-Maya JJ, Aguilar-Romero F, Jaramillo-Meza L, Trigo FJ. Prevalence of Mannheimia haemolytica isolated from bovine nasal exudates and associated factors, in dairy farms in the North-Central of Mexico. J Anim Vet Adv 2007;6(3):404-409.

14. Jaramillo-Arango $\mathrm{C}$, Hernández-Castro $\mathrm{R}$, Suárez-Güemes $\mathrm{F}$, Martínez-Maya JJ, Aguilar-Romero F, Jaramillo-Meza L, Trigo FJ . Characterization of Mannheimia spp. strains isolated from bovine nasal exudates and factors associated to isolates, in dairy farms in the Central Valley of Mexico. Res Vet Sci 2007;84:7-13.

15. Pitcher DG, Saunders NA, Owen RJ. Rapid extraction of bacterial genomic DNA with guanidium thiocyanate. Lett Appl Microbiol 1989 8:151-158.

16. Brosius J, Ullrich A, Raker MA, Gray A, Dull TJ , Gutell RR, Noller HF. Construction and fine mapping of recombinant plasmid containing the $r n B$ ribosomal RNA operon of $E$. coli. Plasmid 1981;6:112-118.

17. Kodjo A, Villard L, Bizet Ch, Martel J L, Sanchis R, Borges E, Gauthier $D$, Maurin F, Richard Y. Pulse-field electrophoresis is more efficient than ribotyping and random amplified polymorphic DNA analysis in discrimination of Pasteurella haemolytica strains. J Clin Microbiol 1999; 37(2):380-385

18. Blackall PJ, Angen O, Fegan N, Blackall LL, Mutters R, Bisgaard M. Characterisation of a novel Mannheimia sp from Australian feedlot cattle. Aust Vet J 2001;79(9):634-639.

19. Snipes KP, Hirsh DC, Kasten RW, Hansen LM, Hird DW, Carpenter TE, McCapes RH. Use of an rRNA probe and restriction endonuclease analysis to fingerprint Pasteurella multocida isolated from turkeys and wildlife. J Clin Microbiol 1989;27(8):1847-1853.

20. Snipes KP, Kasten RW, Wild MA, Miller MW, J essup DA, Silflow RL, et al. Using ribosomal RNA gene restriction patterns in distinguishing isolates of Pasteurella haemolytica from bighorn sheep (Ovis canadiensis). J Wildl Dis 1992;28(3):347-354

21. Saxena MK, Kumar AA, Chaudhari P, Shivachandra SB, Singh VP, Sharma B. Ribotyping of Indian isolates of Pasteurella multocida based on 165 and $23 S$ rRNA genes. Vet Res Commun 2005;29:527535.

22. Angen O, Caugant DA, Olsen JE, Bisgaard M. Genotypic relationships among strains classified under the (Pasteurella) haemolytica-complex as indicated by ribotyping and multilocus enzyme electrophoresis. Zentralb Bakteriol 1997;286:333-354.

23. Blackall PJ, Fegan N, Chew GTI, Hampson DJ. Population structure and diversity of avian isolates of Pasteurella multocida from Australia. Microbiology 1998; 144:279-289.

24. DeRosa DC, Mechor GD, Staats JJ, Chengappa M, Shryock TR. Comparison of Pasteurella spp. simultaneously isolated from nasal and transtracheal swabs from cattle with clinical signs of bovine respiratory disease. J Clin Microbiol 2000;38(1):327-332.

25. Sarafian SK, Woods TC, Knapp JS, Swaminathan B, Morse SA Molecular characterization of of Haemophilus ducreyi by ribosomal DNA fingerprinting. J Clin Microbiol 1991;29:1949-1954. 